\title{
Demo Abstract: Towards a Wireless Building Management System with Minimum Change to the Building Protocols
}

\author{
Qinghua Luo, Hang-yat Lam, Dan Wang \\ The Hong Kong Polytechnic University \\ \{csqluo, cshylam, csdwang\}@ comp.polyu.edu.hk
}

\author{
Daniel Wai-tin Chan \\ Nanchang University \\ chanwaitin.daniel@gmail.com
}

\author{
Yu Peng, Xiyuan Peng \\ Harbin Institute of Technology \\ \{pengyu,pxy\}@hit.edu.cn
}

\begin{abstract}
We develop a wireless system that can (partially) substitute the wired infrastructure underlying Building Automation and Control network (BACnet) of the Building Management System (BMS). There exists effort (e.g., from ZigBee alliance) to develop wireless components for BMS, while they build from bottom up which may lead to change and re-standardization of a new BACnet protocol. There are entirely new infrastructure proposed (e.g., sMAP) to collect physical information. These schemes require a longer time to be adopted. Our system does not need any modification of BACnet. We believe that our design experience is useful for other applications where wired infrastructure and a complete set of upper layer protocols exist; and it is now considered cost-effective to convert the system to (partially) wireless.

Our experience shows that a direct replacement of wires to wireless links does not work. There are a number of difficulties, including a strict time constraint for a frame to exist before the system consider it as outdated. Our solution is to propose an asynchronous-response framework so as to keep the control flow of the upper layer protocol intact. In the demo we will 1) bring the physical devices and explain our system on site and 2) show a video of a real world experiment of our system.
\end{abstract}

\section{INTRODUCTION}

After the introduction and development of wireless sensor networks, the research community has proposed many new applications in the past decade. Recently, researchers have found that it is also fruitful to apply wireless sensor networks to existing applications to replace or partially replace the wired infrastructure. One example is structural health monitoring (SHM) using wireless sensor networks [3]. The wireless feature has great advantages ranging from a cheaper price, to easy system deployment and modification. The onboard processing power, i.e., the smart sensors, can also be used to make the systems more intelligent. Compared to proposing a new application, where their demands are yet to be justified, making existing applications wireless has easyto-see benefit and thus attract many attentions.

Recently we are trying to develop a (partially) wireless building management systems (BMS). The wireless system is flexible. When there needs room re-configuration (e.g., room partitioning), room facility modifications (e.g., light, air-conditioning), etc, substantial cost savings are expected.

One important problem is that existing system usually has a complete set of upper layer protocols developed and even standardized. For example, for BMS, there is Building Automation and Control network (BACnet) that specifies the protocol of the interaction of the sensing devices (e.g., lighting, heating, ventilation and air conditioning, etc), the direct digital controllers (DDC, i.e., the data "relays") and the operation centers of a building. BACnet is developed, standardized and assumed-to-be on top of a wired network.

There are efforts to develop a new physical information collection framework from scratch, e.g., sMAP [2], which can be used for BMS. ZigBee Alliance is more specifically trying to support BACnet using ZigBee communication [1]. Starting from a new wireless bottom layer, we can expect to see new wireless devices (e.g., wireless DDC) and new BACnet standards. Nevertheless, these conversions will take time to finalize and efforts to handle legacy BMS systems.

We ask a different question: can we convert the BMS into wireless, without modification of BACnet (this also means that we should utilize existing hardware with nonintruding extension). Note that we do not say that our scheme substitutes the efforts towards system re-design and upper layer protocols re-standardization. We believe that they are orthogonal and can work in collaboration.

\section{BMS, BACNET AND DESIGN BRIEFING}

Our experience shows that a direct replacement of wires to wireless links does not work. We first briefly introduce the architecture of BMS and BACnet (see Fig. 1). The BMS act as the brain of buildings in controlling and monitoring the mechanical and electrical equipments. In BMS, physical data are recorded by the sensing devices and these sensing devices are connected by the DDCs. The DDCs form the hardware backbone of BMS. The DDCs are classified as system DDCs (usually more powerful) and common DDCs. The physical connection of the DDCs is RS-485, a standard for physical layer data communication that is widely used in buildings, industry control, automation etc. On top of RS-485, there is an MS/TP (Master Slave/Token Passing) protocol. The system DDCs are connected to the operational center using Ethernet. The software element of BMS is BACnet, standardized by ASHRAE. BACnet defines the interaction behavior of the BMS devices. 


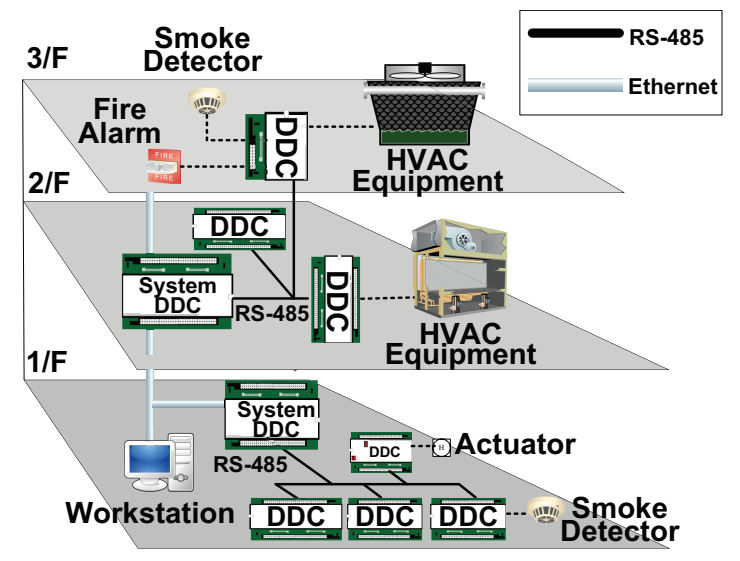

Figure 1: The BMS architecture

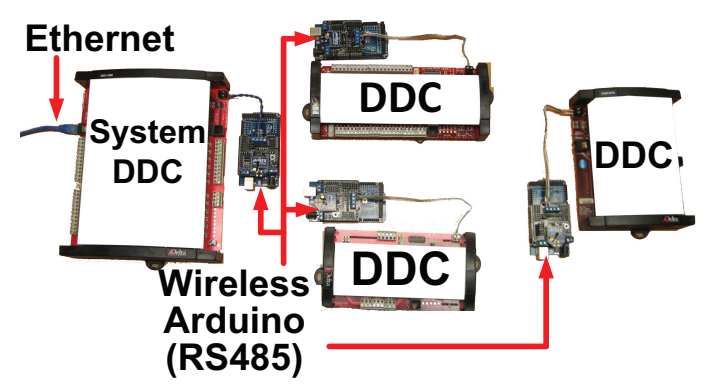

Figure 2: The wireless Arduino connected with DDCs

We focus on transforming the MS/TP DDC connections into wireless communication to improve cost-efficiency and flexibility. The Ethernet DDC connections are less flexible and the wires can be better planned ahead.

Clearly, the transmission of wireless links is slower and more unstable than the wired links. This has two consequences. First, the data throughput from the application layer at certain time may exceed the wireless link capacity. A combined usage of storage and scheduling is needed. Second, for each frame, there is a constraint on delay time. More specifically, if this frame is not received within a certain amount of time, it is considered expired. ${ }^{1}$ In MS/TP, this is 10bit time. This can be easily achieved by a wired link, yet a ZigBee wireless link can only support 9600bit time; and this cannot be improved by increasing bandwidth.

We first equipped the DDCs with wireless Arduino Sensors (Fig. 2). This is non-intruding as Arduino simply reads from RS-485. Our key proposal is to develop a novel asynchronous-response framework. The intuition is as follows. Call the DDC that initiates a query the q-DDC, the sensor attached to it the q-Sensor; and the receiving sensor and receiving DDC $\mathrm{r}-\mathrm{DDC}$ and $\mathrm{r}$-Sensor. After receiving a query from the q-DDC, the q-Sensor sends this query to

\footnotetext{
${ }^{1}$ This is a very common design to keep the system away from lingering around frames/packets. For example, in Internet routing, there is a maximum hop number constraint for each packet. A packet should be dropped if the number of hops exceeds this number.
}

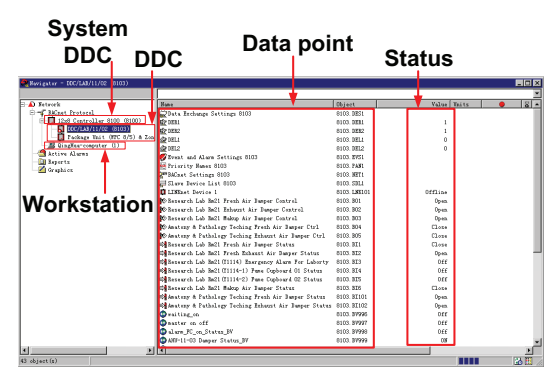

Figure 3: The GUI software for controlling DDCs

the r-Sensor. r-Sensor will send the request to r-DDC and respond to the q-Sensor. To meet the delay constraint, the q-Sensor can send a response to the q-DDC asynchronous to the request sent/received from r-Sensor. Note that we only use valid MS/TP protocol commands. Due to space limitation, we cannot give the details of the implementation of this idea. Asynchronous-response can maintain the control flow of upper layer protocols intact. Third, we have a modular design to prioritize and schedule data transmission in case of link quality and throughput variation. Unfortunately, we do not have the space to describe the details either.

\section{Demo Description}

The demo has three parts. First, we will bring and distribute leaflets that have our detailed design.

Second, we will bring DDCs and show experiments on site (i.e., Fig. 2). Fig. 3 shows the GUI (this is used in the operation center of the building) to control the sensing devices through DDCs. In the experiment, we will 1) use this software to impose changes of the sensing devices (which incurs large data traffic) and 2) turn the readings of individual sensing devices (which incurs small traffic). All these valid operations will go through BACnet which rides on top of our wireless system. The true data traffic will be shown to the audience (note that even there is no operation from the BMS and no data frames, the BACnet protocol still imposes control frames to keep the connectivity of the DDCs and the sensing devices).

Second, for people passing around without an initial point to ask questions, we will bring a video that continuously run in the back end. The video will show the functions of our system and also show a deployment experience in The Hong Kong Polytechnic University. An initial version of this video can be found in [4]. We will bring an advance version with a complete real world experiment.

\section{REFERENCES}

[1] BACnet Unplugged ZigBee and BACnet Connect, ASHRAE Journal 2008, p.42-46, http://www.bacnet.org/Bibliography/AJ-6-2008.pdf

[2] S. Dawson-Haggerty, X. Jiang, G. Tolle, J. Ortiz, and David Culler, "sMAP: a Simple Measurement and Actuation Profile for Physical Information", in Proc. ACM SenSys'10, Zurich, Nov. 2010.

[3] B. Li, D. Wang, F. Wang, and Y. Q. Ni, "High Quality Sensor Placement for SHM Systems: Refocusing on Application Demands", in Proc. IEEE INFOCOM'10, San Diego, CA, Mar. 2010.

[4] Available at http://www4.comp.polyu.edu.hk/ csdwang/Projects/wBACnet.htm 